\title{
The muon g-2 and dark matter in the MSSM at 100 TeV
}

\author{
A. Kobakhidze, ${ }^{a}$ M. Talia ${ }^{* a}$ and L. Wu ${ }^{a b}$ \\ ${ }^{a}$ ARC Centre of Excellence for Particle Physics at the Terascale, \\ School of Physics, The University of Sydney, NSW 2006, Australia \\ ${ }^{b}$ Department of Physics and Institute of Theoretical Physics, \\ Nanjing Normal University, Nanjing, Jiangsu 210023, China \\ E-mail: archil.kobakhidzedsydney • edu.au,

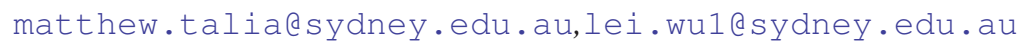

\begin{abstract}
We study the muon g-2 and neutralino dark matter as explained by the MSSM where the squarks and 3rd generation sleptons are decoupled. Particularly, we focus on constraints from current and future dark matter experiments such as PandaX-II and LUX-2016 as well as current bounds from collider searches. Using the constraints on the MSSM from the muon g-2 and DM searches, we study constraints from multilepton + MET searches at $8 \mathrm{TeV}$ LHC, and the prospects for searches at $100 \mathrm{TeV}$ proton-proton collision energies.
\end{abstract}

The European Physical Society Conference on High Energy Physics

5-12 July, 2017

Venice

${ }^{*}$ Speaker. 


\section{Foreword}

Please refer to [1] for more details, particularly concerning the detailed calculation of the $(g-2)_{\mu}$ and collider constraints.

\section{Introduction}

Weak-scale supersymmetry (SUSY) has long been the dominant paradigm for new particle physics. The minimal supersymmetric standard model (MSSM) not only provides an elegant solution to the hierarchy problem but also may successfully explain the $(g-2)_{\mu}$ anomaly $[2,3,4,5,6,7,8,9,10]$. The recently measured value for $\Delta a_{\mu}^{\mathrm{Exp}-\mathrm{SM}} \equiv a_{\mu}^{\mathrm{Exp}}-a_{\mu}^{\mathrm{SM}}[11]$,

$$
\Delta a_{\mu}^{\mathrm{Exp}-\mathrm{SM}}=\left\{\begin{array}{l}
(28.7 \pm 8.0) \times 10^{-10}[12] \\
(26.1 \pm 8.0) \times 10^{-10}[13]
\end{array}\right.
$$

are more than $3 \sigma$ away from the SM prediction, which includes improved QED [14] and electroweak [15] contributions. The upcoming experiments at NBL will measure the $(g-2)_{\mu}$ with a precision of $0.14 \mathrm{ppm}$ [16], which would potentially allow a $5 \sigma$ discovery of new physics through such measurements. In the MSSM, the most significant contribution to $a_{\mu} \equiv(g-2)_{\mu} / 2$ is due to the one-loop diagrams involving the smuon $\widetilde{\mu}$, muon sneutrino $\widetilde{v}_{\mu}$, neutralinos $\widetilde{\chi}^{0}$ and charginos $\tilde{\chi}^{ \pm}$. The one-loop contribution to $a_{\mu}$ arises if there is a chirality flip between incoming and outgoing external muon lines, which may be induced through the $L-R$ mixing in the smuon sector or the SUSY Yukawa couplings of Higgsinos to muon and $\widetilde{\mu}$ or $\widetilde{v}_{\mu}$. Therefore, these contributions to $a_{\mu}$ are typically proportional to $m_{\mu}^{2} / M_{S U S Y}^{2}$. Thus, to generate the sizable contributions to $a_{\mu}$, the SUSY scale $M_{S U S Y}$ encapsulating slepton and electroweakino masses has to be around $O(100)$ $\mathrm{GeV}$. So, the detection of light sleptons and electroweakinos will provide a test for the MSSM solution to the $(g-2)_{\mu}$ problem.

The one-loop corrections mainly rely on the bino/wino masses $M_{1,2}$, the Higgsino mass $\mu$, the left- and right-smuon mass parameters, $M_{\widetilde{\mu}_{L}, \widetilde{\mu}_{R}}$, and the ratio of the two Higgs vacuum expectation values, $\tan \beta$. They have a weak dependence on the second generation trilinear coupling $A_{\mu}$. In the limit of large $\tan \beta$, when all the mass scales are roughly of the same order of $M_{S U S Y}$, the contributions can be approximately written as

$$
\begin{aligned}
& a_{\mu}^{\widetilde{\chi}^{ \pm}} \simeq \frac{m_{\mu}^{2} g_{2}^{2}}{32 \pi^{2} M_{S U S Y}^{2}} \tan \beta \\
& a_{\mu}^{\widetilde{\chi}^{0}} \simeq \frac{m_{\mu}^{2}}{192 \pi^{2} M_{S U S Y}^{2}}\left(g_{1}^{2}-g_{2}^{2}\right) \tan \beta .
\end{aligned}
$$

The detailed dependence of $a_{\mu}$ on the five relevant mass parameters $M_{1,2}, \mu, M_{\widetilde{\mu}_{L}, \widetilde{\mu}_{R}}$ and $\tan \beta$ is complicated. For two-loop corrections, it should be noted that if the squark masses (or masses of the first or third generation slepton) become large, the SUSY contributions to $a_{\mu}$ do not decouple but are logarithmically enhanced. Depending on the mass pattern, a positive or negative correction of $\mathrm{O}(10 \%)$ for squark masses in the few $\mathrm{TeV}$ region can be obtained, see Ref. [20]. 


\section{Constraints on the MSSM Explanation of $(g-2)_{\mu}$}

In the following, we numerically calculate $\Delta a_{\mu}$ by using the FeynHiggs -2.12 .0 [21] package and scan the relevant MSSM parameter space:

$$
\begin{array}{r}
10<\tan \beta<50, \quad-2 \mathrm{TeV}<M_{1}, M_{2}<2 \mathrm{TeV}, \\
-2 \mathrm{TeV}<\mu<2 \mathrm{TeV}, \quad 0.1 \mathrm{TeV}<m_{\widetilde{l}_{L}}, m_{\widetilde{l}_{R}}<2 \mathrm{TeV} .
\end{array}
$$

where we have the subscript $\ell=e, \mu$. Due to the small effects on $a_{\mu}$, the slepton trilinear parameters of the first two generation are assumed as $A_{\ell}=0$. We also decouple the stau sector by setting the soft stau mass parameters $m_{\tilde{\tau}_{L}}=m_{\tilde{\tau}_{R}}=5 \mathrm{TeV}$ and trilinear parameter $A_{\tau}=0$. So the stau will not contribute to the trilepton signals in our simulations. To satisfy the $125 \mathrm{GeV}$ Higgs mass within a 2 $\mathrm{GeV}$ deviation, we vary the stop trilinear parameter in the range $\left|A_{t}\right|<5 \mathrm{TeV}$ and set the stop soft masses at $5 \mathrm{TeV}$. We require the mixing parameter $\left|X_{t} / M_{S}\right|<2$ to avoid the charge/colour-breaking minima [22]. We additionally calculate the Higgs mass and the rest of the sparticle masses with FeynHiggs-2.12.0 [21].

\subsection{LEP and Higgs data}

In our scan, we also consider the following experimental bounds:

- LEP: the direct searches for the slepton and chargino at LEP produce the lower mass limits on the first two generation sleptons and lightest chargino [23]:

$$
\begin{aligned}
m_{\widetilde{l}_{L}}, m_{\widetilde{l}_{R}}>100 \mathrm{GeV} \quad(l=e, \mu) \\
m_{\widetilde{\chi}_{1}^{ \pm}}>105 \mathrm{GeV}
\end{aligned}
$$

- Higgs data: the exclusion limits at 95\% CL from the experimental cross sections from higgs searches at LEP, Tevatron and LHC are examined by using HiggsBounds -4 . 2 . 1 [24].

- We require the lightest neutralino $\widetilde{\chi}_{1}^{0}$ as the LSP and $m_{\widetilde{\chi}_{1}^{0}}>30 \mathrm{GeV}$ to be consistent with the bound on light MSSM neutralino dark matter [25].

In Fig. 1, we present the dependence of $\Delta a_{\mu}$ on the masses of neutralinos $\left(\widetilde{\chi}_{1,2}^{0}\right)$, charginos $\left(\widetilde{\chi}_{1,2}^{ \pm}\right)$and smuons $\left(\widetilde{\mu}_{1,2}\right)$. Within the scan ranges of Eq. 2.1 , We find that the $\widetilde{\chi}^{ \pm}-\widetilde{v}_{\mu}$ loop dominates over the $\tilde{\chi}^{0}-\tilde{\mu}$ loop. A sizable SUSY contribution to $a_{\mu}$ can be obtained, if $M_{1}, M_{2}$ and $\mu$ have the same sign and $\widetilde{\chi}_{1,2}^{0}$ and $\widetilde{\chi}_{1}^{ \pm}$have a sizable higgsino, wino or both components with large $\tan \beta$. The explanation of $\Delta a_{\mu}$ within a $2 \sigma$ range requires $m_{\widetilde{\chi}_{1}^{0}}<1.0 \mathrm{TeV}$ and $m_{\widetilde{\mu}_{1}}<1.03 \mathrm{TeV}{ }^{1}$. However, a higgsino or wino-like LSP typically cannot satisfy the constraints of the dark matter relic density and are constrained using data from direct detection experiments.

\footnotetext{
${ }^{1}$ It should be noted that if the higgsino mass parameter $\mu$ is large enough, the $g-2$ anomaly may be explained through the bino-smuon loop contribution, due to the large smuon left-right mixing [26]. But such a large $\mu$ scenario is disfavored by the vacuum stability [26], the naturalness [27] and are highly constrained by the dark matter relic density [28].
} 

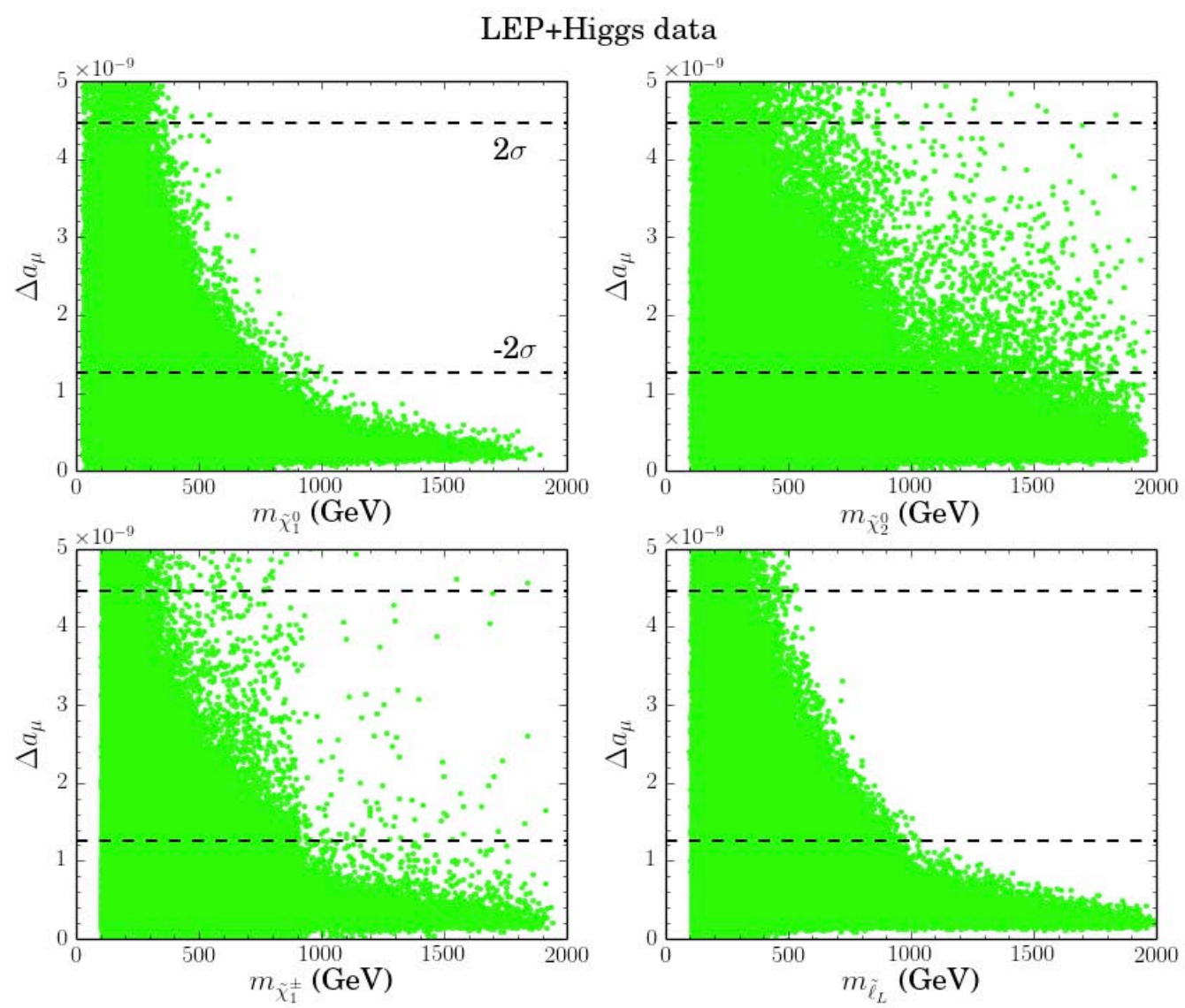

Figure 1: Scatter plot on the plane of $\Delta a_{\mu}$ and sparticle masses. Green circles satisfy the constraints from LEP and LHC Higgs data. The dashed lines represent the $2 \sigma$ band on $\Delta a_{\mu}$ given by Eq.(1.1).

\subsection{DM relic density and direct detection experiments}

Next, we confront the MSSM explanation of $(g-2)_{\mu}$ with the various dark matter experiments. We use MicrOmegas -4.2 .3 [29] to calculate the dark matter relic density $\Omega h^{2}$ and the spin-independent neutralino scattering cross sections with nuclei, denoted as $\sigma^{S I}$. It should be noted that the thermal relic abundance of the light higgsino or wino-like neutralino dark matter is typically low due to the large annihilation rate in the early universe. This leads to the standard thermally produced WIMP dark matter being under-abundant. In order to have the correct relic density, several alternatives have been proposed, such as choosing the axion-higgsino admixture as a dark matter candidate [30]. So we rescale the scattering cross section $\sigma^{S I}$ by a factor of $\left(\Omega h^{2} / \Omega_{\text {Planck }} h^{2}\right)$, where $\Omega_{\text {Planck }} h^{2}=0.112 \pm 0.006$ is the relic density measured by Planck satellite [31].

In Fig. 2, we show the neutralino dark matter relic density $\Omega h^{2}$ (left) and the spin-independent neutralino-nucleon scattering cross section $\sigma^{S I}$ (right). All samples satisfy the LEP, Higgs data and $(g-2)_{\mu}$ within $2 \sigma$. In the left panel of Fig. 2 , it can be seen that there are an amount of samples above the $3 \sigma$ upper bound of the Planck relic density measurement. Those samples are bino-like and annihilate to the SM particles very slowly, which leads to an overabundance of dark matter in the universe. On the other hand, there are two dips around $M_{Z}$ and $M_{h}$, respectively, where $\widetilde{\chi}_{1}^{0} \widetilde{\chi}_{1}^{0}$ 

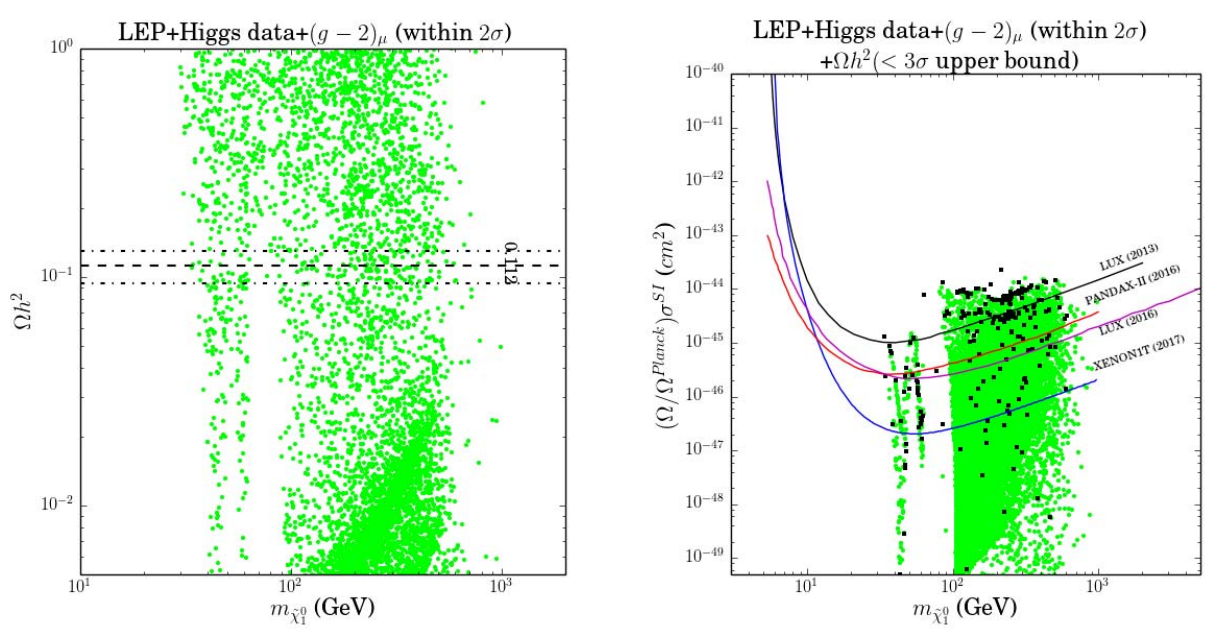

Figure 2: The neutralino dark matter relic density $\Omega h^{2}$ (left) and the spin-independent neutralino-nucleon scattering cross section $\sigma^{S I}$ (right). The dashed line is the PLANCK central value and the dashed-dotted lines are corresponding $3 \sigma$ bands. The exclusion limits on the $\sigma^{S I}$ from LUX (2013) [32] (black line), LUX (2016) (magenta line) [34], PandaX-II (red line) [33], and XENON1T (2017) projected [37] (blue line). Green circles satisfy the LEP, Higgs data and $2 \sigma$ bound of $(g-2)_{\mu}$ (left) and $3 \sigma$ upper bound of $\Omega h^{2}$, while the black squares further require $\Omega h^{2}$ within $3 \sigma$ range.

can efficiently annihilate through the resonance effect. When the LSP higgsino or wino component dominates, the annihilation cross section of $\widetilde{\chi}_{1}^{0} \widetilde{\chi}_{1}^{0}$ is small so that the relic density is less than the $3 \sigma$ lower bound of the Planck value. A mixed LSP with a certain higgsino or wino fraction [28] can be reconciled with the measured relic abundance $\Omega h^{2}$ within the $3 \sigma$ range. In the right panel of Fig. 2, we project the samples that satisfy $3 \sigma$ upper bound $\Omega_{\text {Planck }} h^{2}$ on the plane of $\sigma^{S I}$ versus $m_{\widetilde{\chi}_{1}^{0}}$.

A significant portion of the parameter space where the LSP has a sizable higgsino or wino component is excluded by the recent PandaX-II [33] and LUX data [34]. The samples with nearly pure higgsino or wino LSPs escape experimental constraints due to the large reduction in the DM abundance. We also find some samples with the correct DM relic density (within $3 \sigma$ ) and satisfying the LUX constraints. These samples can be placed in two categories. The smaller portion of samples belong to the so called MSSM blind-spot region of parameters [35, 36] where the LSP coupling to the Higgs boson is so small that the DM-nucleon scattering cross section is highly suppressed. The sfermions and other heavy higgs bosons are decoupled for these particular samples. The second case is that the bino-like LSPs coannihilate with the sleptons. The scattering cross section of the bino-like LSP with the nucleon can be small to avoid the LUX bound. The future XENON1T (2017) experiment [37] will further cover the these parameter space.

\subsection{LHC 8 TeV collider search}

Given the great progress of LHC experiments, we recast the results of searching for $2 \ell+E_{T}$ and $3 \ell+E_{T}$ signatures at LHC- $8 \mathrm{TeV}$. We focus on $8 \mathrm{TeV}$ data. In fact, most of dedicated analyses at $13 \mathrm{TeV}$ are either preliminary $[44,45,46]$ or do not provide stronger constraints in general due to the still small luminosity [47]. The main processes contributing to $2 \ell+E_{T}$ events can arise from 


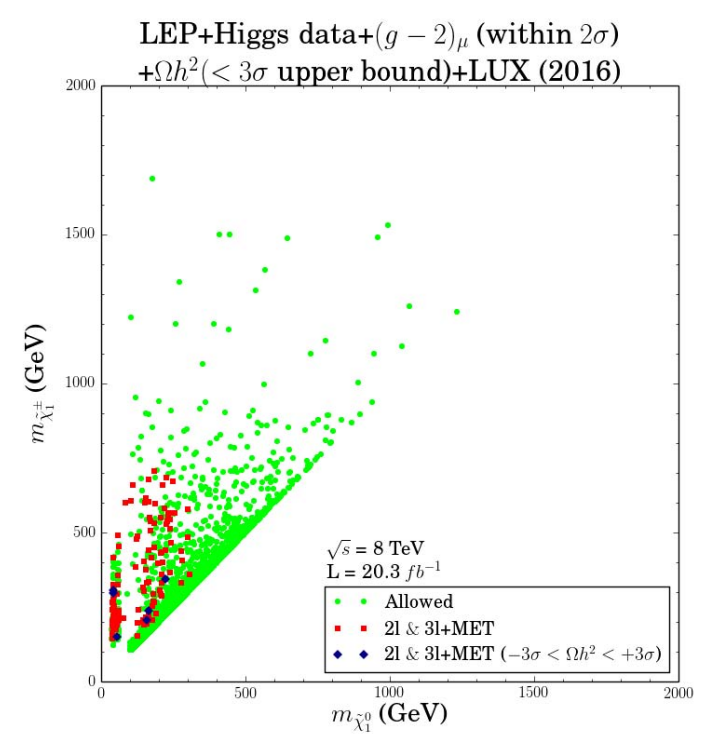

Figure 3: Exclusion limits from LHC Run-1 dilepton and trilepton events. All samples satisfy the LEP, Higgs data, $3 \sigma$ upper bound of the dark matter relic density, LUX 2016 and $(g-2)_{\mu}$ within the $2 \sigma$. Red squares $\left(\Omega h^{2}<+3 \sigma\right)$ and blue diamonds $\left(-3 \sigma<\Omega h^{2}<+3 \sigma\right)$ are excluded by $2 \ell+E_{T}^{\prime}$ and $3 \ell+E_{T}^{\prime}$ events.

the production of sleptons pair and charginos:

$$
p p \rightarrow \widetilde{\ell}^{+} \tilde{\ell}^{-}, \widetilde{\chi}_{1}^{+} \widetilde{\chi}_{1}^{-}
$$

with the subsequent decays to leptons through direct slepton decay, or chargino decay through sleptons, sneutrinos or $\mathrm{W}$ bosons. Meanwhile, $3 \ell+E_{T}$ events mainly come from the associated production of chargino and neutralino:

$$
p p \rightarrow \widetilde{\chi}_{i}^{0} \widetilde{\chi}_{j}^{ \pm}
$$

where $i=2,3,4$ and $j=1,2$. They then decay in two different ways: through sleptons/sneutrinos or through SM gauge bosons.

We use SPheno-3.3.8 [38] to produce the SLHA file to employ in MadGraph5_aMC@NLO [39] and generate the parton level signal events. Then the events are showered and hadronized by PYTHIA [40]. The detector effects are included by using the tuned Delphes [41]. Fast Jet [42] is used to cluster jets with the anti- $k_{t}$ algorithm [43]. We recast the ATLAS dilepton [17] and trilepton [19] analyses by using CheckMATE-1.2.2 [48]. We include the NLO correction effects in the production of $\widetilde{\ell}^{ \pm} \widetilde{\ell}^{\mp}, \widetilde{\chi}_{i}^{ \pm} \widetilde{\chi}_{i}^{\mp}$ and $\widetilde{\chi}_{i}^{0} \widetilde{\chi}_{j}^{ \pm}$productions by multiplying a $K$-factor 1.3 [49]. The main SM backgrounds include $W Z, Z Z$ and $t t V(V=W, Z)$. To estimate the exclusion limit, we define the ratio $r=\max \left(N_{S, i} / S_{o b s, i}^{95 \%}\right)$, where $N_{S, i}$ and $S_{o b s, i}^{95 \%}$ are the event numbers of the signal for $i$-th signal region and the corresponding observed 95\% C.L. upper limit, respectively. The max is over all signal regions defined in the analysis. We conclude that a sample is excluded at $95 \%$ C.L., if $r>1$.

In Fig. 3, we recast the LHC Run-1 dilepton and trilepton exclusion limits on the plane of $m_{\widetilde{\chi}_{1}^{ \pm}}$ and $m_{\widetilde{\chi}_{1}^{0}}$. All samples satisfy the LEP, Higgs data, $3 \sigma$ upper bound of relic density, LUX 2016 and 
$(g-2)_{\mu}$ within $2 \sigma$ range. Red squares $\left(\Omega h^{2}<+3 \sigma\right)$ and blue diamonds $\left(-3 \sigma<\Omega h^{2}<+3 \sigma\right)$ are excluded by $2 \ell+E_{T}^{\prime}$ and $3 \ell+E_{T}^{\prime}$ events. In Fig. 3, we can see that a portion of samples in $\tilde{\chi}_{1}^{ \pm}<710 \mathrm{GeV}$ and $\widetilde{\chi}_{1}^{0}<300 \mathrm{GeV}$ can be excluded. A bulk of samples in the parameter space with $\widetilde{\chi}_{1}^{0}$ being higgsino or wino-like can not be covered because of the small mass difference between $\widetilde{\chi}_{1}^{ \pm}$ and $\widetilde{\chi}_{1}^{0}$. Such a region may be accessed by the monojet(-like) or the VBF production at HL-LHC $[50,51,52,53,54,55,56]$.

\section{Prospects at a $100 \mathrm{TeV}$ Collider}

To hunt for new fundamental particles, a $100 \mathrm{TeV} p p$ collider has been under discussion in recent years, which will allow us to probe the new physics scale roughly an order of magnitude higher than we can possibly reach with the LHC [57]. In this section, we estimate the prospects of probing the MSSM explanation of the $(g-2)_{\mu}$ anomaly by extrapolating the above $8 \mathrm{TeV}$ trilepton analysis to a $100 \mathrm{TeV} p p$ collider. For each allowed sample above, we use the most sensitive signal region in $8 \mathrm{TeV}$ analysis and simply assume the same detection efficiency in the $100 \mathrm{TeV}$ analysis. We rescale the signal $(S)$ and background $(B)$ events by the following ratio:

$$
N^{100 \mathrm{TeV}}=\left(\sigma^{100 \mathrm{TeV}} / \sigma^{8 \mathrm{TeV}}\right)\left(3000 \mathrm{fb}^{-1} / 20.3 \mathrm{fb}^{-1}\right) N^{8 \mathrm{TeV}}
$$

Such a treatment can be considered as a preliminary theoretical estimation. The optimized analysis strategy may be achieved once the details of the collider environment is known. To obtain the expected exclusion limits, we use the following equation,

$$
\left.\frac{S}{\sqrt{B+\left(\beta_{\text {sys }} B\right)^{2}}} \geq 2 \quad \text { [Excluded }\right]
$$

where the factor $\beta_{\text {sys }}$ parameterizes the systematic uncertainty. In Fig. 4, we can see that when $\beta_{s y s}=0.1$, a majority of samples allowed by $(g-2)_{\mu}$ in the parameter space with $\widetilde{\chi}_{1}^{0}<530 \mathrm{GeV}$ and $\widetilde{\chi}_{1}^{ \pm}<940 \mathrm{GeV}$ can be excluded. Such a range will be extended to $\widetilde{\chi}_{1}^{0}<710 \mathrm{GeV}$ and $\widetilde{\chi}_{1}^{ \pm}<940$ $\mathrm{GeV}$, if $\beta_{\text {sys }}=0$.

It should be noted that the region that satisfies the DM relic density within the $3 \sigma$ range through the $Z$ or $h$ resonant annihilation in the blind spots can be covered by searching for trilepton events from $\tilde{\chi}_{2}^{0} \tilde{\chi}_{1}^{+}$associated production at a $100 \mathrm{TeV} p p$ collider. The samples that are beyond future sensitivity of this trilepton search and the DM direct detections are either a higgsino/wino-like LSP with a compressed mass spectrum or bino-like with a large slepton co-annihilation cross section. Such compressed regions may be covered by the monojet(-like) searches at a $100 \mathrm{TeV} p p$ collider [58].

\section{Conclusion}

Under the constraints of Higgs data, dark matter relic density, PandaX-II/LUX-2016 experiments and LHC-8 TeV searches for dilepton/trilepton events, we find the Planck data and the recent PandaX-II/LUX data can significantly exclude the MSSM parameter space satisfying $(g-2)_{\mu}$, which will be further excluded by the upcoming XENON-1T (2017) experiment. We also find that most of our surviving samples that satisfy DM relic density within $3 \sigma$ range through $Z$ or $h$ resonant 


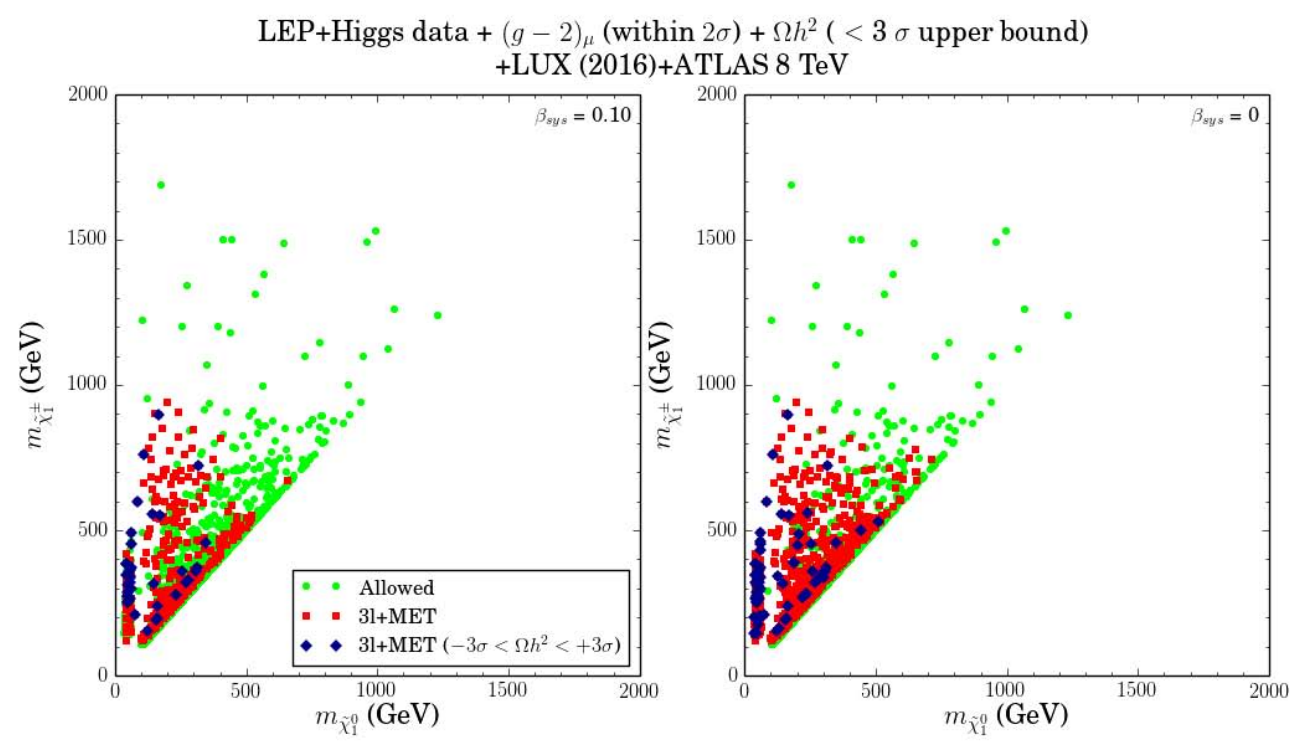

Figure 4: Same as Fig. 3, but for expected exclusion limit at a $100 \mathrm{TeV} p p$ collider with the luminosity of $3000 \mathrm{fb}^{-1}$. Red squares $\left(\Omega h^{2}<+3 \sigma\right)$ and blue diamonds $-3 \sigma<\Omega h^{2}<+3 \sigma$ are excluded by searching for $3 \ell+$ MET events. The systematic uncertainty $\beta_{\text {sys }}$ is taken as 0.1 and 0 , respectively.

effect can be covered by searching for trilepton events from $\tilde{\chi}_{2}^{0} \tilde{\chi}_{1}^{+}$associated production at a 100 $\mathrm{TeV} p p$ collider. While the samples that are beyond the future sensitivity of this trilepton search and DM direct detections are either higgsino/wino-like LSP or bino-wino/slepton co-annihilation. Such compressed regions may be covered by the monojet(-like) searches at a future $100 \mathrm{TeV} p p$ collider.

\section{References}

[1] Kobakhidze, A., Talia, M., Wu L., Phys. Rev. D 95, 055023 (2017)

[2] T. Moroi, Phys. Rev. D 53, 6565 (1996) Erratum: [Phys. Rev. D 56, 4424 (1997)].

[3] S. P. Martin and J. D. Wells, Phys. Rev. D 64, 035003 (2001).

[4] D. Stöckinger, J. Phys. G 34, R45 (2007).

[5] G. -C. Cho, K. Hagiwara, Y. Matsumoto and D. Nomura, JHEP 1111, 068 (2011).

[6] M. Endo, K. Hamaguchi, S. Iwamoto and T. Yoshinaga, JHEP 1401, 123 (2014).

[7] M. Ibe, T. T. Yanagida and N. Yokozaki, JHEP 1308, 067 (2013).

[8] M. A. Ajaib, B. Dutta, T. Ghosh, I. Gogoladze and Q. Shafi, Phys. Rev. D 92, no. 7, 075033 (2015).

[9] M. Badziak, Z. Lalak, M. Lewicki, M. Olechowski and S. Pokorski, JHEP 1503 (2015) 003.

[10] P. Athron et al., Eur. Phys. J. C 76, no. 2, 62 (2016).

[11] G. W. Bennett et al. [Muon g-2 Collaboration], Phys. Rev. D 73, 072003 (2006).

[12] M. Davier, A. Hoecker, B. Malaescu and Z. Zhang, Eur. Phys. J. C 71, 1515 (2011) Erratum: [Eur. Phys. J. C 72, 1874 (2012)]. 
[13] K. Hagiwara, R. Liao, A. D. Martin, D. Nomura and T. Teubner, J. Phys. G 38, 085003 (2011).

[14] T. Aoyama, M. Hayakawa, T. Kinoshita and M. Nio, Phys. Rev. Lett. 109, 111808 (2012).

[15] C. Gnendiger, D. StÃúckinger and H. StÃúckinger-Kim, Phys. Rev. D 88, 053005 (2013).

[16] G. Venanzoni [Fermilab E989 Collaboration], Nucl. Part. Phys. Proc. 273-275, 584 (2016).

[17] The ATLAS collaboration [ATLAS Collaboration], ATLAS-CONF-2013-049.

[18] V. Khachatryan et al. [CMS Collaboration], Eur. Phys. J. C 74, no. 9, 3036 (2014).

[19] G. Aad et al. [ATLAS Collaboration], JHEP 1404, 169 (2014).

[20] H. Fargnoli, C. Gnendiger, S. PaÃßehr, D. StÃúckinger and H. StÃúckinger-Kim, JHEP 1402, 070 (2014).

[21] S. Heinemeyer, W. Hollik and G. Weiglein, Comput. Phys. Commun. 124, 76 (2000); Eur. Phys. J. C 9, 343 (1999).

[22] U. Chattopadhyay and A. Dey, JHEP 1411, 161 (2014).

[23] K. A. Olive et al. [Particle Data Group Collaboration], Chin. Phys. C 38, 090001 (2014).

[24] P. Bechtle et al., Comput. Phys. Commun. 182, 2605 (2011); Comput. Phys. Commun. 181, 138 (2010).

[25] L. Calibbi, J. M. Lindert, T. Ota and Y. Takanishi, JHEP 1411, 106 (2014).

[26] M. Endo, K. Hamaguchi, T. Kitahara and T. Yoshinaga, JHEP 1311, 013 (2013) doi:10.1007/JHEP11(2013)013 [arXiv:1309.3065 [hep-ph]].

[27] R. Arnowitt and P. Nath, Phys. Rev. D 46, 3981 (1992).

[28] N. Arkani-Hamed, A. Delgado and G. F. Giudice, Nucl. Phys. B 741, 108 (2006) doi:10.1016/j.nuclphysb.2006.02.010 [hep-ph/0601041].

[29] G. Belanger et al., Comput. Phys. Commun. 182, 842 (2011).

[30] H. Baer, A. Lessa, S. Rajagopalan and W. Sreethawong, JCAP 1106 (2011) 031.

[31] P. A. R. Ade et al. [Planck Collaboration], arXiv:1502.01589 [astro-ph.CO].

[32] D. S. Akerib et al. [LUX Collaboration], Phys. Rev. Lett. 112 (2014) 091303.

[33] A. Tan et al. [PandaX-II Collaboration], Phys. Rev. Lett. 117, no. 12, 121303 (2016) doi:10.1103/PhysRevLett.117.121303 [arXiv:1607.07400 [hep-ex]].

[34] D. S. Akerib et al., arXiv:1608.07648 [astro-ph.CO].

[35] C. Cheung, L. J. Hall, D. Pinner and J. T. Ruderman, JHEP 1305, 100 (2013) doi:10.1007/JHEP05(2013)100 [arXiv:1211.4873 [hep-ph]].

[36] T. Han, F. Kling, S. Su and Y. Wu, [arXiv:1612.02387 [hep-ph]].

[37] XENON1T Collaboration, E. Aprile, et al., arXiv:1206.6288.

[38] W. Porod, Comput. Phys. Commun. 153, 275 (2003) doi:10.1016/S0010-4655(03)00222-4 [hep-ph/0301101].

[39] J. Alwall et al., JHEP 1407, 079 (2014).

[40] T. Sjostrand, S. Mrenna and P. Z. Skands, JHEP 0605, 026 (2006). 
[41] J. de Favereau, et al., arXiv:1307.6346 [hep-ex].

[42] M. Cacciari, G. P. Salam and G. Soyez, Eur. Phys. J. C 72, 1896 (2012) [arXiv:1111.6097 [hep-ph]].

[43] M. Cacciari, G. P. Salam and G. Soyez, JHEP 0804, 063 (2008).

[44] ATLAS-CONF-2016-075

[45] CMS-PAS-SUS-16-024.

[46] CMS-PAS-SUS-16-022

[47] G. Aad et al. [ATLAS Collaboration], Eur. Phys. J. C 76, no. 5, 259 (2016) doi:10.1140/epjc/s10052-016-4095-8 [arXiv:1602.09058 [hep-ex]].

[48] M. Drees et al., Comput. Phys. Commun. 187, 227 (2014).

[49] W. Beenakker, R. Hopker and M. Spira, hep-ph/9611232.

[50] A. Barr and J. Scoville, JHEP 1504, 147 (2015).

[51] P. Schwaller and J. Zurita, JHEP 1403, 060 (2014).

[52] C. Han, A. Kobakhidze, N. Liu, A. Saavedra, L. Wu and J. M. Yang, JHEP 1402, 049 (2014).

[53] H. Baer, A. Mustafayev and X. Tata, Phys. Rev. D 89, no. 5, 055007 (2014).

[54] C. Han, L. Wu, J. M. Yang, M. Zhang and Y. Zhang, Phys. Rev. D 91, 055030 (2015).

[55] B. Dutta et al., Phys. Rev. D 91, no. 5, 055025 (2015).

[56] B. Dutta et al., Phys. Rev. D 90, no. 9, 095022 (2014).

[57] N. Arkani-Hamed, T. Han, M. Mangano and L. T. Wang, arXiv:1511.06495 [hep-ph].

[58] M. Low and L. T. Wang, JHEP 1408, 161 (2014). 